\section{Chemotherapiewirksamkeit von Tumorbiologie abhängig}

Die meisten Patienten mit einem Glioblastom sind über 60 Jahre alt. In einer randomisierten Studie wurde nun untersucht, welches die optimale palliative Behandlung für Patienten ist, die keine Radiochemotherapie tolerieren.

$\mathrm{R}_{\mathrm{n}}^{\mathrm{a}}$ andomisiert erhielten 342 Patienten mit neu diagnostiziertem Glioblastom entweder Temozolomid $\left(200 \mathrm{mg} / \mathrm{m}^{2}\right.$ an den Tagen $1-5$, alle 28 Tage, bis zu sechs Zyklen), eine hypofraktionierte Radiotherapie (34 Gy in zehn 3,4-GyFraktionen über zwei Wochen) oder eine Standardradiotherapie (60 Gy in 2,0-GyFraktionen über sechs Wochen).

Beim primären Endpunkt medianes Gesamtüberleben war Temozolomid der Standardbestrahlung signifikant überlegen (8,3 vs. 6,0 Monate, Hazard Ratio [HR] 0,70; $\mathrm{p}=0,01)$. Zwischen hypofraktionierter und Standardbestrahlung gab es keinen signifikanten Unterschied. Auch Temozolomid und die hypofraktionierte Radiotherapie lieferten vergleichbare Ergebnisse ( 8,4 vs. 7,4 Monate, HR $0,82 ; \mathrm{p}=0,12)$.

Allerdings waren die Resultate abhängig vom Alter der Patienten: Bei Studienteilnehmern im Alter von 60 bis 70 Jahren war das Überleben unter allen drei Therapieregimes vergleichbar. Über 70-jährige Patienten lebten aber sowohl mit Temozolomid als auch mit der hypofraktionierten Radiotherapie länger als mit der Standardbestrahlung (Temozolomid vs. Standardbestrahlung: HR 0,35; p $<0,0001$; hypofraktionierte vs. Standardbestrahlung: HR 0,59; $\mathrm{p}=0,02$ ). Bei Patienten, die mit Temozolomid behandelt wurden, hatte die Methylierung des MGMT-Promotors einen signifikanten Einfluss auf das Überleben (mit vs. ohne Methylierung: 9,7 vs. 6,8 Monate, HR 0,$56 ; \mathrm{p}=0,02$ ).

Neutropenien und Thrombozytopenien waren die häufigsten Grad-3/4-Nebenwirkungen in der Temozolomid-Gruppe. Infektionen der Grade 3-5 traten bei 18 Patienten aus allen Gruppen auf. Zwei Patienten erlitten tödliche Infektionen (einer unter Temozolomid und einer mit Standardradiotherapie). Ein weiterer $\mathrm{Pa}$ tient aus der Temozolomid-Gruppe starb an gastrointestinalen Blutungen aufgrund postoperativer Komplikationen.
Fazit: Die Standardbestrahlung ging mit schlechterem Überleben einher, vor allem bei über 70-Jährigen. Sowohl Temozolomid als auch die hypofraktionierte Radiotherapie kommen als Standardtherapieoptionen bei älteren Patienten mit Glioblastom in Betracht. Dabei könnte es sinnvoll sein, den MGMT-Methylierungsstatus in die Therapieentscheidung mit einzubeziehen.

Judith Neumaier

Malmström A et al. Temozolomide versus standard 6-week radiotherapy versus hypofractionated radiotherapy in patients older than 60 years with glioblastoma: The Nordic randomised, phase 3 trial. Lancet Oncol. 2012;13(9):916-26.

Kommentar von Prof. Schlegel: Die vorliegende Studie führt sich mit dem selbstbewussten Anspruch ein, einen neuen "Standard" für die Therapie älterer Patienten mit Glioblastom zu definieren. Dies erscheint vor dem Hintergrund relevanter methodischer Probleme ambitioniert:

_ Über mehr als neun Jahre wurden in 28 (!) beteiligten Zentren weniger als 400 Patienten rekrutiert, wobei zwischenzeitlich publizierte Ergebnisse den Therapiestandard für Subgruppen der Patienten änderten bzw. neu definierten [Stupp R et al. N Engl J Med. 2005;352(10): 987-96; Keime-Guibert F et al. N Engl J Med. 2007; 356(15):1527-35]. Dadurch wurde die Änderung der unteren Altersgrenze für den Studieneinschluss während der Studienphase erforderlich.

_ Die Studie ist nicht gepowert für eine bestimmte Hypothese; eine Fallzahlabschätzung vor Beginn der Studie wurde nicht durchgeführt, wenngleich im Text angemerkt ist, man hätte für die Signifikanz einer Differenz von $10 \%$ bezüglich des primären Endpunktes insgesamt 480 Patienten benötigt.

_ Der wesentliche Parameter für die Wirksamkeit einer zu untersuchenden Primärtherapie, das progressionsfreie Überleben, wurde nicht bestimmt.

_ Dass eine bei $28 \%$ der Patienten inkomplett durchgeführte Therapie (Standard-
Strahlentherapie) einer bioäquivalenten, aber bei $95 \%$ der Patienten komplett durchgeführten Therapie (hypofraktionierte Strahlentherapie mit 10 x 3,4 Gy) unterlegen ist, überrascht nicht.

\section{„Vor Therapiebeginn \\ MGMT-Methylierungsstatus bestimmen"}

Der für die tägliche Praxis wichtigste Befund ist der prädiktive Wert eines positiven $\mathrm{O}^{6}$-Methylguanin-Methyltransferase(MGMT)-Promotor-Methylierungsstatus bezüglich des Ansprechens auf eine Chemotherapie mit Temozolomid: Bei Durchführung einer alleinigen Strahlentherapie spielte der Methylierungsstatus keine Rolle für das Gesamtüberleben. Die mit Temozolomid allein behandelten Patienten zeigten jedoch bei positivem Methylierungsstatus ein signifikant besseres Gesamtüberleben als bei negativem Methylierungsstatus: 9,7 vs. 6,8 Monate Gesamtüberleben; für die bestrahlten Patienten betrug dieser Wert für beide Gruppen 8,2 vs. 7,0 Monate.

So ergeben sich folgende praktische Konsequenzen:

_ Bei Diagnose eines malignen Glioms beim älteren Menschen mit Therapiewunsch und Therapieindikation muss die MGMTPromotor-Methylierung analysiert werden.

_ Bei negativer MGMT-Promotor-Methylierung ist eine ausschließliche Strahlentherapie der erweiterten Tumorregion indiziert. (Ein etabliertes hypofraktioniertes Protokoll mit 15 x 2,7 Gy für Glioblastompatienten ab 60 Jahre gibt es bereits [Roa W et al. J Clin Oncol. 2004;22(9):1583-8]

- Bei positiver MGMT-Promotor-Methylierung kann eine ausschließliche Chemotherapie mit Temozolomid in Standarddosis durchgeführt werden. Dies kann durch den gleichsinnigen Befund der "NOA08Studie" [Wick W et al. Lancet Oncol. 2012; 13(7):707-15] begründet werden, auch wenn in dieser Studie ein intensiviertes Temozolomid-Protokoll benutzt wurde.

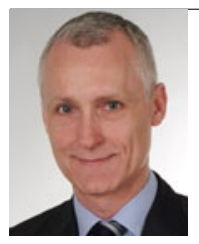

Prof. Dr. med.

Uwe Schlegel

Klinik für Neurologie Knappschaftskrankenhaus Bochum uwe.schlegel@kk-bochum. de 